\title{
Enthalten gewisse Organe des Körpers physiologischer Weise Arsen?
}

Von

\author{
Dr. C. Hödlmoser,
} klinischem Assistenten.

(Aus der III. medic. Klinik in Wien.)

(Der Redaction zugegangen am 10. Juli 1901.)

Ende des Jahres 1899 veröffentlichte Armand Gautier eine Mittheilung, welche vollauf berechtigt war, grosses Aufsehen hervorzurufen. Gautier ${ }^{1}$ ) kündigte nämlich an, dass er in gewissen Organen der Thiere und Menschen schon physiologischer Weise Arsen gefunden habe. Gautier war auf folgendem Wege zu dieser Entdeckung gelangt. Jod und Arsen sind zwei bei Krankheiten der Schilddrüse gewissermassen specifische Medicamente. Jod und Arsen finden sich vielfach in der Natur zusammen vor, so in den Algen, in vielen Mineralwässern. Da nun die nach Erkrankungen der Schilddrüse auftretenden schweren Anämien gemäss den Erfahrungen Gautier's besonders auf Arsen reagiren, und da die Schilddrüse nach den seither vielfach bestätigten Forschungen Baumann's einen beträchtlichen Gehalt an Jod aufweist, so schloss Gautier, dass möglicher Weise auch die Schilddrüse und vielleicht andere Organe physiologischer Weise Arsen enthalten könnten. Als Gautier an seine Nachforschungen über den physiologischen Arsengehalt ging, konnte er sich allerdings nicht verhehlen, dass zahlreiche Erfahrungen gegen

1) A. Ga utier, Sur l'existence normale de l'arsenic chez les animaux et sa localisation en certains organes. Bullet. d. l'académie de médecine 1899. 5. December. Siehe auch Comptes rendues. 1899, II, 2, S. 929. 
diese Annahme von vornherein sprächen, in erster Linie der Umstand, dass ja bei zahlreichen gerichtlich-chemischen Untersuchungen, welche auf den Nachweis des Arsens gerichtet waren, die Abwesenheit des Arsens beim normalen Individuum constatirt wurde. Als Momente, welche diese negativen Befunde erklären könnten, erscheinen Gautier folgende: zunächst der Umstand, dass nach seinen Erfahrungen bei den gewöhnlich gehandhabten Methoden des Arsennachweises die sehr geringen physiologischen Arsenmengen, um welche es sich handeln könnte, verloren gingen und andererseits das Moment, dass allerdings in den meisten und zwar den in gerichtlichen Fällen gewöhnlich untersuchten Organen normaler Weise Arsen vollständig fehle, wohl aber nach der von Gautier angegebenen Methode sich in einigen wenigen Organen Arsen nachweisen lasse. Nur die Schilddrüse, in geringeren Mengen die Thymusdrüse, die Haut und das Gehirn enthielten beim Menschen und verschiedenen Thieren normaler Weise Arsen, während die anderen Organe, wie Leber, Milz etc. davon frei befunden wurden. Gautier hatte damals seine Untersuchungen auf Schilddrüsen vom Menschen, vom Hunde, vom Schweine und vom Schafe ausgedehnt, ferner zum Vergleiche auf die Lebern vom Schafe, Rind, Hunde und Schweine, die Milz des Hundes und des Rindes, die Nieren des Schweines, das Muskelfleisch des Hundes, menschliche Testikel und endlich defibrinirtes Schweineblut. Die Methode, welche Gautier anwendet und welche er im Wesentlichen schon im Jahre $1876^{1}$ ) publicirt hatte, ist entsprechend den von ihm in den Comptes rendues ${ }^{2}$ ) publicirten Modificationen in den Hauptzügen folgende: $100 \mathrm{~g}$ des frischen Organes werden mit $30-60 \mathrm{~g}$ reiner Salpetersäure unter Zusatz von $1 \mathrm{~g}$ Schwefelsäure und unter vorsichtigem Erwärmen bis zur vollständigen Eindickung und Verflüssigung

1) A. Gautier, Sur la recherche et le dosage de l'arsenic dans les matières animales. Annales de Chimie et de Physique. $5^{0}$ série, t. VIII, pag. 384 .

2) A. Gautier; Recherche et dosage des très petites quantités d'arsenic dans les organes. Comptes rendues 1899, II, 2, S. 936. 
behandelt; in die erkaltete Flüssigkeit werden dann 8-10 g reiner Schwefelsäure eingetragen, und das Ganze von Neuem erhitzt; hierauf unter langsamem wiederholten Zusatze geringer Mengen Salpetersäure die Oxydation der organischen Substanz bewirkt, bis das Aufhören des Entstehens rothbrauner Dämpfe das Ende der 0xydation anzeigt. Es wird hierauf bis zum Entweichen dichter, weisser Schwelsäuredämpfe erhitzt, wobei in der Schale nur noch eine bräunlich-schwarze Flüssigkeit zurückbleibt, welche sich bei der Siedetemperatur der Schwefelsäure auch noch lange Zeit nicht entfärbt. Gautier gibt selbst an, dass in vielen Fällen, besonders auch bei der Schilddrüse, es selır lange Zeit brauche, bis die Oxydation erreicht sei. Die bei dieser Procedur resultirende bräunliche Flüssigkeit: wird hierauf neuerlich mit etwas Schwefelsäure versetzt und sodann unter Umschwenken langsam in ungefähr $600-700 \mathrm{ccm}$. Wasser geleert, die Schale mit Wasser nachgewaschen. Von. dem sich nunmehr absetzenden fein vertheilten Niederschlage wird abfiltrirt und in das bräunliche Filtrat wird nach Zusatz von 1-2 ccm. schwefliger Säure durch mehrere Stunden unter Erwärmen Schwefelwasserstoff eingeleitet.

Der sich bildende Niederschlag wird nach zwölfstündigem Absetzenlassen auf einem Filter gesammelt, gewaschen und mitsammt dem Filter in einer Schale mit ammoniakhaltigem Wasser durch 40 Minuten bei einer Temperatur von beiläufig $40-50^{\circ}$ behandelt, dann neuerlich filtrirt; das Filtrat wird eingedampft und der Rückstand mit einer Mischung von Schwefelsäure und Salpetersäure oxydirt. Zur Vertreibung der letzten Spuren von Salpetersäure wird sodann die Flüssigkeit bis zum Auftreten dichter, weisser Dämpfe erhitzt; hierauf mit Wasser verdünnt und in den Marsh'schen Apparat eingetragen.

Für die. Handhabung des Marsh'schen Apparates hatte Gautier schon im Jahre 1876 genaue Vorschriften angegeben, namentlich bezüglich der Länge, in welcher das Rohr erhitzt werden muss. Der Marsh'sche Apparat muss nach Gautier's Vorschrift durch drei Stunden im Gang sein, nach welcher Zëit das ganze in der eingetragenen Flüssigkeit enthaltene Arsen als Arsenspiegel abgeschieden ist. Durch Wägung des 
Arsenspiegels erfolgt die quantitative Bestimmung des Arsens.

Schon 1876 hatte Gautier angegeben, dass seine Methode gegenüber allen anderen den Vorzug besitze, dass durch sie minimale Spuren von Arsen nachgewiesen werden könnten, welche nach den übrigen gebräuchlichen Methoden sich dem Nachweise entziehen. Er behauptet, durch seine Methode ein hundertstel Milligramm Arsen in $200 \mathrm{~g}$ Substanz nachweisen zu können. Ich werde auf Gautier's Methode noch später, bei Besprechung meiner eigenen diesbezüglichen Erfahrungen, näher eingehen.

Die Resultate der Eingangs erwähnten Untersuchungen Gautier's waren folgende: Schon $\mathbf{5 , 2} \mathrm{g}$ frischer Hundeschilddrüse, von zwei Thieren herstammend, enthielten eine minimale Spur von Arsen. $45 \mathrm{~g}$ frischer Schweineschilddrüse gaben einen Spiegel, welcher, verglichen mit einer Reihe von Spiegeln bekannten Gewichtes, eine Menge vorstellte, die ungefähr $0,7 \mathrm{mg}$ Arsen auf $1 \mathrm{~kg}$ frischer Drüse entsprach, fast $3 \mathrm{mg}$ auf $1 \mathrm{~kg}$ getrockneter Drüse.

Aus $100 \mathrm{~g}$ frischer Schafschilddrüse gewann Gautier einen Spiegel von $0,05 \mathrm{mg}$. Mit noch verbesserter Methode stellte dann Gautier an $\mathbf{1 2 7} \mathrm{g}$ menschlicher Schilddrüsen eine Untersuchung an, und zwar an Schilddrüsen von Individuen, welche während ihrer Krankheit fast gar kein Medicament und namentlich kein Arsen zu sich genommen hatten. Die Schilddrüsen stammten von sechs Individuen, welche an folgenden Krankheiten gelitten hatten: 1 . Erysipel und Pneumonie, 2. Wirbelfractur, 3. tbc. Meningitis, 4. intestinale Obstruction, 5. Magenkrebs, 6. Pleuritis mit Ascites. Aus diesen $127 \mathrm{~g}$ gewann Gautier einen Arsenspiegel im Gewichte von $\mathbf{0 , 9 5} \mathrm{mg}$. Diese Untersuchungen wurden von Gautier sorgfältig wiederholt unter steten vergleichenden Versuchen mit der arsenfreien Leber vom Schafe.

Im Februar $1900^{1}$ ) erschien eine weitere Mittheilung

1) A. Gautier, Localisation, élimination et origines de l'arsenic chez les animaux. Bullet. d. l'académie de médecine. 1900. 6. Fevrier. Siehe auch Comptes rendues. 1900. I, 1, S. 284. 
Gautier's über neuerliche Versuche, welche sich auf eine Reihe von Organen erstreckten und folgendes Resultat hatten:

1. Arsenhaltige Organe. Arsengehalt in $100 \mathrm{~g}$ frischer Substanz. Schilddrüse $0,7 \check{~ m g}$.

Brustdrüse $0,13 \mathrm{mg}$.

Gehirn, wechselnde Quantität oder negativer Befund.

Thymus, sehr deutliche, aber quantitativ nicht bestimmbare Menge.

Fell, Haare, Hornsubstanzen

Haut

Milch

Knochen

Der Reihe nach abnehmende Spuren.

Diese Ergebnisse sind nach Gautier als vorläufige zu betrachten.

Als nicht arsenhaltig erwiesen sich dagegen alle übrigen Organe, von denen Gautier sowohl beim Menschen als auch bei verschiedenen Thieren eine ganze Reihe untersucht hatte. Besonders möchte ich hervorheben, dass sich die Leber, wenigstens bei Verwendung von 100-200 g frischen Organes zur Untersuchung, als absolut arsenfrei erwies.

Auch das Blut, Harn und Faeces waren vollständig arsenfrei.

Eine weitere Untersuchungsreihe, in derselben Mittheilung veröffentlicht, hatte die Durchforschung verschiedener Nahrungsmittel auf ihren Arsengehalt zum Gegenstande. Gautier hatte schon in seiner ersten Mittheilung erwähnt, dass einige pflanzliche Nahrungsmittel nach den Untersuchungen von Stein geringe Mengen von Arsen enthalten, so der Kohl, die Kartoffel, die Kohlrübe, alles Nahrungsmittel, in denen Arsen mit dem Jod und Eisen zusammen vorkommt. Es seien dies namentlich die Nahrungsmittel, welche auf den Kalklagern der tertiären Gesteinsformen wachsen. Brot, Fleisch, Eier, Fisch erwiesen sich als frei von Arsen.

Obwohl nun das Blut sich als frei von Arsen erwies, so konnte Gautier natürlich die Annahme nicht von der Hand weisen, dass das Arsen sich doch zeitweise, wenn auch in minimalen nicht nachweisbaren Spuren im Blute finden müsse und die Schilddrüse die besondere Fähigkeit besitze, das Arsen aufzuhäufen, um daraus die nach Gautier's An- 
schauung für die richtige Function der Thyreoidea nothwendige Substanz zu bilden. Es drängte sich nun Gautier die Frage auf, in welcher Form das Arsen in der Schilddrüse existire; er dachte sofort daran, dass möglicher Weise, bei dem vielfach analogen Verhalten des Phosphors mit dem Arsen, die Zellkerne in ihrem Nuclein das Arsen enthielten. Dafür sprach ihm auch die bereits wiederholt gemachte Beobachtung, dass metallische Substanzen, und überhaupt Körper von höherem Molekulargewicht, wenn sie ins Blut eindringen, von den Zellkernen gebunden werden.

Um diese Frage zu klären, unterwarf Gautier $100 \mathrm{~g}$ Schilddrüse vom Schafe unter Zusatz von 1/2 g Pepsin und Verdauungssalzsäure der Verdauung. Die hierbei entstehende Flüssigkeit wurde filtrirt und die am Filter zurückbleibenden Nucleine wurden in sehr verdünntem Ammoniak gelöst. Die nunmehr nach seiner Methode auf Arsen geprüften Nucleine fand Gautier deutlich arsenhaltig, während die Peptone arsenfrei waren.

Daraus schloss er, dass normaler Weise in der Schilddrüse und den anderen von ihm früher bezeichneten Organen Arsen in der Form von "Arsennucleinen» vorhanden sei, welche ähnlich wie die gewöhnlichen Phosphornucleine zusammengesetzt wären und welche im Haushalte des Organismus eine wichtige Rolle spielen müssen, indem gerade bei Erkrankungen der Schilddrüse die Arsentherapie von Vortheil sei, und da endlich bei Erkrankungen der Schilddrüse gerade die Organe, in welchen sich das Arsen nachweisen lässt, am meisten zu leiden pflegen.

An diese Mittheilung knüpfte der Verfasser Betrachtungen darüber, dass, ebenso wie Arsen und Jod in relativ geringen Mengen eine so wichtige Function besitzen, möglicher Weise auch noch andere Elemente, welche man mit den heutigen verfeinerten Methoden in geringen Spuren nachweisen könne, im thierischen Organismus vielleicht eine grosse Rolle spielen und vielleicht durch Substitution ihnen verwandter Elemente die Wirksamkeit der verschiedenen Organe modificiren können.

Im August 1900 erschien dann noch eine dritte wichtige 
Mittheilung ${ }^{1}$ ) Gautier's, betitelt: La fonction menstruelle et le rut des animaux; Rôle de l'arsenic dans l'économie. Ich kann hier nur das Wichtigste aus den geistvollen Hypothesen und Betrachtungen, welche Gautier in dieser Mittheilung vorbringt, hervorheben.

Nach Gautier bestehen zwischen den Functionen des Genitalapparates, der Schilddrüse und dem Wachsthum der Haare, Nägel, Horngebilde etc. sichere Beziehungen, wenn diese auch in ihrem Wesen noch nicht geklärt sind. Eine Reihe von Momenten spricht nach Gautier dafür, dass unter Kakodylwirkung das Haar der behandelten Frauen stärker zu wachsen beginnt, die Haut von Epheliden, Pigmentationen $u$. s. w. gereinigt wird und die Menstruation in etwas kürzeren Intervallen und dabei regelmässiger eintrete. Auch der Jodgebrauch rufe ähnliche Wirkungen hervor. Es könne daher vielleicht möglich sein, dass durch die Horngebilde sowohl wie auch durch die menstruelle Blutung die Ausscheidung des vorher assimilirten Arsens und des Jods erfolge. Auf das menstruelle Blut richtete daher Gautier seine Untersuchungen.

Von sechs Frauen fing Gautier das Menstrualblut in arsenfreier und vorher gewogener Watte auf. Die Watte mit dem Blute wurde wieder gewogen und darin nach Arsen gesucht. Folgendes waren die dabei gewonnenen Resultate:

\begin{tabular}{|c|c|c|c|}
\hline & $\begin{array}{c}\text { Menge des } \\
\text { Blutes }\end{array}$ & $\begin{array}{c}\text { Menge des } \\
\text { Arsens }\end{array}$ & $\begin{array}{l}\text { Dosen } \\
\text { pro Kilo } \\
\text { Blut }\end{array}$ \\
\hline I. & $195 \mathrm{~g}$ & $0,06 \mathrm{mg}$ & $0,33 \mathrm{mg}$ \\
\hline II. & 367 , & 0,06 . & 0,17 , \\
\hline III. & 60 & 0,02 & 0,33 \\
\hline IV. & 46 & 0,015 » & 0,32 , \\
\hline V. & 120 & 0,03 , & 0,25 \\
\hline
\end{tabular}

1) A. Gautier, La fonction menstruelle et le rut des animaux; Rôle de l'arsenic dans l'économie. Bullet. d. l'académie de médecine. 1900. 7. August. 
Daher im Mittel auf $1 \mathrm{~kg}$ Blut 0,28 mg Arsen.

In einem sechsten Falle, betreffend eine Frau mit auffallend blassem Menstrualblut, konnte keine Spur Arsen nachgewiesen werden, woraus der Verfasser den Schluss zieht, dass derartig anämisches Menstruationsblut kein Arsen enthalte.

Gautier's Assistent konnte für das Jod feststellen, dass das Menstrualblut eineinhalbmal so viel Jod enthalte, als das normale.

Bemerkenswerth ist die Thatsache, dass die Schilddrüse mitunter vor Eintritt der Menstruation an Volumen zunimmt, dass sie häufig zur Zeit der Gravidität eine besonders starke Entwicklung zeigt. Anderseits beobachtet man bei an Myxödem leidenden Kranken eine Atrophie der Genitalien, die unter Einwirkung von Schilddrüsensaft sich wieder besser zu entwickeln beginnen. Bei der Frau werden durch das Menstrualblut, durch die Haut und ihre Adnexe Arsen und Jod ausgeschieden.

Um aber die Ausscheidung des Arsenüberschusses bei den männlichen Wesen zu erklären, recurrirt Gautier zunächst auf die Thatsache, dass bei vielen Thiergattungen zur Brunftzeit das Abfallen eines Theiles der Behaarung des Körpers stattfindet, der im Herbste sich wieder herstellt. Es liesse sich diese Thatsache nicht einfach durch die Einwirkung der warmen resp. kalten Jahreszeit erklären, sondern der Grund dafür liegt nach Gautier's Auffassung darin, dass die arsenhaltigen Nucleoproteide zunächst zur Ernährung der Haut und der Horngebilde verwendet werden, während am Beginne der Brunftzeit die Nucleoproteide gegen die Genitalorgane geleitet werden, die nunmehr mangelhaft versorgten Haare ausfallen und die Haut häufig von Eczem ergriffen wird.

Beim männlichen Individuum des Menschen, welchem keine Brunftzeit eigen ist, erfolge durch das Wachsen der Nägel und des Bartes, sowie durch die continuirliche Abschuppung der Epidermis eine langsame Ausscheidung des Arsens, während die Frau, deren Haut glatter ist und geringere Exfoliation zeige, die keinen Bartwuchs habe und deren Haare langsam bis zur Erreichung der Pubertät wachse, dann aber stehen bleibe, eben durch die Menstruation ihren Arsenüberschuss ausscheide. 
Ausser diesen und noch anderen der Physiologie entnommenen Belegen zieht Gautier auch mehrere Facten aus der Pathologie zur Stütze seiner Ansicht heran, z. B. das Auftreten von Hautkrankheiten bei Frauen während der Schwangerschaft, zu welcher Zeit das Arsen und damit für die Ernährung der Haut wichtige Substanzen gegen den Genitaltract hin abgeleitet werden; ferner den Umstand, dass bei Tuberculösen, bei welchen Gautier eine starke Abnahme des physiologischen Arsengehaltes der Schilddrüse beobachtet hat, häufig krankhafte Veränderungen der Haut, sowie Amenorrhöe und Dysmenorrhöe auftreten, die sich auf Verabreichung von Kakodylpräparaten, besonders bei gleichzeitigem Zusatze geringer Quantitäten von Jod, bessern.

Da nun die Angabe, dass der thierische Organismus unter normalen Verhältnissen in verschiedenen Organen Arsen enthalten solle, auch mir von der grössten Wichtigkeit schien, so machte ich mich daran, die Untersuchungen Gautier's nachzuprüfen, auch in der weiteren Absicht, die Schilddrüsen bei verschiedenen Krankheiten mit einander bezüglich ihres Arsengehaltes zu vergleichen, sowie besonders. auch durch Experimente am Menschen und Thiere über das Verhalten der Drüse nach Einnahme von Arsen Aufschlüsse zu bekommen.

Ich möchte nun heute über meine bisherigen einschlägigen Ergebnisse berichten, welche sich bis jetzt nicht in Uebereinstimmung mit den Befunden Gautier's befinden.

Ich muss gestehen, dass mir im vorhinein der Befund Gautier's befremdend erschien, da er ja vollständig von dem gewöhnlichen Verhalten von Metallgiften nach ihrer Aufnahme in den Organismus abweicht; für gewöhnlich werden ja Metallgifte nach ihrer Aufnahme in den Organismus in erster Linie in der Leber abgelagert.

Gerade für das Arsen erbrachte E. Ludwig ${ }^{1}$ ) in seiner

1) E. Ludwig, Ueber die Vertheilung des Arsens im thierischen Organismus und Einverleibung von arseniger Säure. Medic. Jahrb., Wien, 1880, 4. Heft.

Hoppe-Seyler's Zeitschrift f. physiol. Chemie. XXXIII. 
1880 publicirten Untersuchungsreihe den unumstösslichen sicheren Beweis, dass die Leber das Hauptdepot des eingeführten Arsens bilde.

E. Ludwig hatte sowohl verschiedene Leichentheile aus sanitätspolizeilich obducirten Leichen mit Arsenvergiftung auf ihren Arsengehalt untersucht, als auch bei einer Reihe von Hunden acute und chronische Arsenvergiftung erzeugt und die Leichentheile derselben qualitativ und quantitativ auf Arsen untersucht. Die Arbeit war besonders mit Rücksicht auf eine von Scolobus off im Laboratorium Gautier's 1876 ausgeführte Arbeit unternommen worden, welche zu dem Resultate gekommen war, dass bei der acuten Arsenvergiftung das Arsen zuerst in das Gehirn und dann rasch in das Rückenmark gelange, und auch bei der chronischen Vergiftung sich in seiner Hauptmenge im Gehirn und Rückenmark localisire, die Leber dagegen und die Muskeln bedeutend geringere Mengen dieses Elementes enthalten. (In einem Falle sogar $40 \mathrm{mal}$ so viel Arsen im Gehirn und Rückenmark als im gleichen Gewichte Muskel und 3 mal so viel als im gleichen Gewichte Leber.) Nach den Untersuchungen von E. Ludwig unterliegt es dagegen keinem Zweifel, dass sowohl bei der acuten als auch bei der chronischen Arsenvergiftung im Gehirne nur geringe Mengen Arsen zu finden seien, dagegen sowohl Leber als Niere einen hohen Arsengehalt aufweisen, letztere besonders in acuten Fällen. Bei chronischen Arsenvergiftungen bleibt, wenn die Einverleibung des Giftes ausgesetzt wird, dieses am längsten in der Leber zurück. In der Leber eines Hundes wurde vierzig Tage nach der letzten Arsenikgabe noch reichlich Arsen aufgefunden, während Gehirn, Knochen und Muskel arsenfrei waren.

Aus meiner eigenen Erfahrung möchte ich noch bezüglich des Bleies hinzufügen, dass ich in mehreren Fällen, in welchen Prof. Kolisko aus charakteristischen Veränderungen der Gefässe am Secirtische die Diagnose einer länger bestandenen Bleieinwirkung gestellt hatte, auch wenn das Blei schon Jahre lang ausgesetzt worden war, noch minimale Spuren von Blei in der Leber nachweisen konnte. 
Ueber den Arsengehalt der Schilddrüsen nach Vergiftungen liegen allerdings keine anderweitigen Arbeiten vor.

Bezüglich des Arsengehaltes von epithelialen $\mathrm{Ge}$ bilden existirt eine Arbeit von E. Schiff, ${ }^{1}$ ) welcher, um Aufschlüsse zu gewinnen, wodurch in dermatologischen Fällen das Arsen wirke, die Haare von Hunden, an welche er Arsen verfüttert hatte, untersuchte und in denselben ganz deutliche Arsenspuren nachweisen konnte, während bei Kontrollthieren die Haare absolut arsenfrei waren.

Ich habe meine Untersuchungen zum grösseren Theile nach der Eingangs geschilderten Methode Gautier's, zum geringeren Theile nach der Methode von E. Ludwig ${ }^{2}$ ) und Zillner, resp. Fresenius und Babo, durchgeführt, nachdem vorher die Reagentien auf Arsenfreiheit geprüft worden waren.

Es wurden dazu Schilddrüsen von Patienten, welche an verschiedenen Krankheiten verstorben waren, verwendet; für die gütige Ueberlassung des dazu nothwendigen Materiales bin ich Herrn Hofrath Prof. Weischselbaum zu bestem Danke verpflichtet. Ich habe in jedem Falle immer eine dem Gewicht der Schilddrüse ungefähr adäquate Menge Leber gleichfalls für die Untersuchung reservirt, um auf diese Weise vergleichende Resultate über den eventuellen Gehalt der Schilddrüse und der Leber an Arsen zu gewinnen. Ich habe zur Analyse immer mehrere Schilddrüsen resp. Lebern vereinigt, um an einer grösseren Menge Substanz zu operiren.

In folgender Tabelle sind die Sectionsdiagnosen der Fälle, sowie die Mengen des verwendeten, nach Gautier's Methode untersuchten Materiales kurz zusammengestellt.

\begin{tabular}{c|c|c|c}
\hline \hline Nr. & D i a g n o s e n & $\begin{array}{c}\text { Schild- } \\
\text { druise }\end{array}$ & Leber \\
\hline 1 & $\begin{array}{c}\text { Fractura claviculae et scapulae, jauchige Pericarditis } \\
2\end{array}$ & $85 \mathrm{~g}$ & $85 \mathrm{~g}$ \\
& $\begin{array}{r}\text { Vitium cordis mit Endocarditis, Myocarditis mit } \\
\text { Pleuritis, Tbc. mult. . . . . . . . . . . }\end{array}$ & 110, & $110 ;$
\end{tabular}

1) E. Schiff, Ueber die Ablagerung von Arsen in den Haaren. -Wien, klin. Wochenschr., 1898, S. 537.

2) E. Ludwig, Medicinische Chemie, 2. Auflage, 1895. 


\begin{tabular}{|c|c|c|c|}
\hline Nr. & Diagnosen & $\begin{array}{l}\text { Schild- } \\
\text { drüse }\end{array}$ & Leber \\
\hline 3 & Prostata hypertrophie, Cystitis, Fettnecrose d.Pancreas & $145 \mathrm{~g}$ & $145 \mathrm{~g}$ \\
\hline 4 & $\begin{array}{l}\text { Tbc. pulmon. (2 Mal) Hämorrhagia cerebri, Myode- } \\
\text { generatio cordis . . . . . . . . . . }\end{array}$ & $200 \gg$ & 200 \\
\hline 5 & Pyämie, Vitium cordis, Tbc. pulmon., Morbus Addisoni & $170 \gg$ & 170 \\
\hline 6 & $\begin{array}{l}\text { Tbc. pulmon. mit Paral progress. Dysenterie, Sepsis } \\
\text { puerperalis . . . . . . . . . . . . }\end{array}$ & $175 »$ & 185 \\
\hline 7 & Bronchiectasie, Gangraena pulmon., Nephritis chronica & 115 & $110 \diamond$ \\
\hline 8 & $\begin{array}{l}\text { Tbc. pulmon., Amyloidose, Tbc. miliar., Tbc. pulmon. } \\
\text { et periton. . . - . . . . . . . . - } \\
\text { Emphysema pulmon., Paralysis progress. und Tbc. }\end{array}$ & $140 *$ & $16 \check{1}$ \\
\hline 9 & Sepsis puerperalis, Anämie . . . . . . . & $130 »$ & 130 \\
\hline
\end{tabular}

Ausser diesen detaillirt angegebenen Untersuchungen habe ich noch ungefähr 8-10 Mal Schilddrüse und Leber untersucht, jedoch ausnahmslos mit negativem Resultate.

Es waren mitunter allerdings kaum wahrnehmbare Spuren eines Arsenspiegels vorhanden, jedoch nicht öfter in der Schilddrüse als in der Leber, und mir ist aus meiner Thätigkeit als Assistent am medicinisch-chemischen Institute in Erinnerung, dass mitunter bei Untersuchung von Leichentheilen ganz minimale Spuren von Arsen gefunden werden, über deren Provenienz man sich schwer Rechenschaft geben kann.

Um nun den Beweis zu erlangen, dass mir nicht bei Ausführung von Gautier's Methode ein grober Fehler unterlief, welcher die negativen Ergebnisse hätte herbeiführen können, führte ich die Methode mit mehreren Proben der Leber eines Paralytikers aus, welchen ich genau bestimmte Mengen von Arsenik zugesetzt hatte.

Es wurden untersucht:

a) $100 \mathrm{~g}$ Leber ohne Zusatz (Kontroll)

b) $100 \gg,+1 \mathrm{ccm}$. einer Arseniklösung, welche

c) $100 \curvearrowright+2,0,000582 \mathrm{~g} \mathrm{As}_{2} \mathrm{O}_{3}$ per

d) $100, \cdots+3, \int$ Cubikcentimeter enthielt.

Es entstanden nun in den drei letzten Bestimmungen intensive Arsenspiegel, der Spiegel in Probe $c$ und $d$ wurde gewogen. Spiegel c, welcher hätte $0,0009 \mathrm{~g}$ wiegen sollen, 
wog 0,0007 $\mathrm{g}$ und Spiegel d, dessen Gewicht hätte $0,001 \mathrm{~g}$ betragen sollen, wog gleichfalls $0,0007 \mathrm{~g}$.

Ich konnte aus diesen Resultaten wohl ersehen, dass mir Mengen, wie sie von Gautier als normal für menschliche Schilddrüsen angegeben wurden, schwerlich hätten entgehen können.

Ich möchte mir bei dieser Gelegenheit einige Bemerkungen zu Gautier's Methode gestatten. Gautier hat angegeben, dass bei Ausführung der gewöhnlichen Methoden, speciell bei Zerstörung der organischen Substanz mit Salzsäure und chlorsaurem Kali, grosse Verluste an Arsen entständen und sich diese letztere Methode daher für den Nachweis sehr geringer Arsenmengen nicht eigne. Beim Erhitzen mit Salzsäure könnten Verluste durch Verflüchtigung von Chlorarsen entstehen und beim Schmelzen mit Soda und Salpeter könnten gleichfalls geringe Spuren von Arsen sich verflüchtigen. Um nun dies Verhalten zu prüfen, habe ich $200 \mathrm{~g}$ Schweinsschilddrüse, welchen $2 \mathrm{ccm}$. der früher erwähnten Arseniklösung zugesetzt waren, nach der Methode von E. Ludwig und Zillner zerstört und auf Arsen geprüft; das Resultat war ein mächtiger Arsenspiegel, welcher sich an Intensität nicht von dem nach Gautier's Methode erhaltenen unterschied. Eine gleichzeitig angestellte Kontrollprobe mit der gleichen Menge Schweinsschilddrüse ohne Zusatz von Arsen ergab ein absolut negatives Resultat.

Die Methode von Gautier hat nach meinen Erfahrungen folgende Nachtheile: 1. es ist ausserordentlich schwierig, den Zeitpunkt $\mathrm{zu}$ erreichen, in welchem bei Zusatz von Salpetersäure keine deutliche $0 x y d a t i o n$ mehr stattfindet, abgesehen davon, dass das fortwährende Entweichen der Salpetersäuredämpfe, auch wenn die Operation unter dem Herde vorgenommen wird, bei wiederholten Untersuchungen sehr quälend wirkt; 2. als einen beträchtlichen Mangel möchte ich auch die Behandlung des nach der Vertreibung des Ammoniaks restirenden Rückstandes nur mit Salpetersäure und Schwefelsäure ohne Oxydation mit kohlensaurem und salpetersaurem Natrium bezeichnen, indem es nach meinen Erfahrungen unmöglich ist, auf diese Weise eine von organischer Substanz vollständig freie Lösung zu erhalten, was zur weiteren Folge hat, dass jenseits der Glühstelle im Marsh- 
schęn Apparate, offenbar infolge der Zersetzung flüchtiger Kohlenwasserstoffe, sich kohlige Massen ablagern können, welche einerseits einen Arsenspiegel vortäuschen, anderseits aber das Gewicht der Spiegel beeinträchtigen können. Ich glaube daher auch, dass den Gewichtsangaben Gautier's, welche durchwegs auf hundertstel Milligramme angegeben sind, obiger Fehler anhaften dürfte.

Was endlich Gautier's Vorschrift betrifft, das Glühen der Röhre auf eine Strecke von $25-30 \mathrm{~cm}$. vorzunehmen, so habe ich mich überzeugt, dass die Arsenmengen, um die es sich hier handelt, auch wenn das Glühen nur an einer kurzen Stelle des Rohres vorgenommen wird, vollständig abgeschieden werden, indem jenseits einer zweiten Glühstelle kein Arsenspiegel mehr entsteht.

Da ich bis Weihnachten vergangenen Jahres nur negative Resultate erhalten hatte, so wandte ich mich schriftlich an Herrn Professor Gautier um Rath; er rieth mir in liebenswürdiger Weise, die Versuche mit Schweins- oder Hammelschilddrüsen, und zwar mit dem durch Pepsinverdauung hergestellten Nuclein fortzusetzen.

Allein auch das Resultat dieser Versuche war negativ, sowohl nach Gautier's Methode unter Anwendung rauchender Salpetersäure, wie er es mir gerathen hatte, als auch nach der Methode: Zerstörung mit rauchender Salzsäure und chlorsaurem Kali.

Ich habe nun mit Rücksicht auf das früher erwähnte Experiment, dass nämlich aus $200 \mathrm{~g}$ Leichentheilen (Schweinsschilddrüsen), mit $1 \mathrm{mg}$ Arsen versetzt, auch bei Anwendung der Methode von Ludwig und Zillner ein ungemein deutlicher Arsenspiegel entsteht, neuerlich eine Reihe menschlicher Schilddrüsen und entsprechende Gewichtsmengen Leber gesammelt und nach diesem Verfahren untersucht.

Es wurden von 15 Individuen, welche an folgenden Krankheiten gestorben waren: 7 an Tuberculose verschiedener Art, 1 an Insufficientia valv. mitralis, 2 an Carcinoma ventriculi, 1 an Emphysema pulmonum und Lebercirrhose, 1 an Ulcus ventriculi mit Peritonitis, 2 an Dementia paralytica und 1 an Herzparalyse, Schilddrüse und entsprechende Menge Leber im 
Gesammtgewichte von je $380 \mathrm{~g}$ auf Arsen verarbeitet, es konnte jedoch weder aus der Schilddrüse noch aus der Leber die leiseste Spur von Arsen gewonnen werden.

Bei dieser Gelegenheit hat sich neuerdings die absolute Reinheit der im Laboratorium für medicinische Chemie $\mathrm{zu}$ gerichtlichen Zwecken verwendeten Reagentien gezeigt, indem trotz fast vierstündigem Gange des Marsh'schen Apparates auch nicht der leiseste Anflug eines Arsenspiegels zu sehen war.

Ein Fall ') aus meiner eigenen Erfahrung dürfte wohl geeignet sein, die Empfindlichkeit der eben genannten Methode, wenn sie unter allen Cautelen vorgenommen wird, zu demonstriren. Ich konnte nämlich in einer Erdprobe, welche mit dem Erbrochenen eines durch Arsen vergifteten Mannes verunreinigt war, obwohl bereits mehrere Tage vergangen waren, seitdem der Vergiftete daselbst erbrochen und obwohl es inzwischen stark geregnet hatte, noch deutlich Arsen nachweisen; eine Untersuchung, welche im Laboratorium Professor Kratschmer's vorgenommen worden war.

Es erhebt sich nun die wichtige Frage, wieso dieser Gegensatz zwischen Gautier's positiven und meinen eigenen negativen. Resultaten $z u$ erklären ist.

Weit entfernt davon, schon heute ein Gautier direkt entgegengesetztes Resultat definitiv auszusprechen, wollte ich nur meine bisherigen, allerdings nicht sehr zur Fortsetzung der Untersuchungen verlockenden Resultate mittheilen und zu anderweitiger Nachprüfung der jedenfalls höchst interessanten Angaben Gautier's auffordern, gerade so wie auch ich die Untersuchungen, und zwar besonders mit Schilddrüsen von Menschen, welche, ohne vorher krank gewesen $z u$ sein, an kurz dauernden Krankheiten gestorben sind, fortsetzen will.

Ich betone dies Letztere namentlich mit Rücksicht auf die allerdings nicht mit Zahlen belegten Angaben Gautier's, dass bei Tuberculösen der Arsengehalt der Schilddrüse beträchtlich abnehme. Da nun ein Theil der von mir untersuchten Schilddrüsen von tuberculösen Individuen stammte,

1) C. Hödlmoser, Ueber einen Fall von Arsenvergiftung. Wien. klin. Wochenschr., 1898, S. 849. 
so könnte eventuell darin ein das Zustandekommen positiver Befunde behinderndes Moment erblickt werden. Aber selbst wenn man die Schilddrüsen Tuberculöser abrechnet, so blieben immerhin noch für jede einzelne meiner Untersuchungen eine genügende Zahl von Schilddrüsen, welche ein positives Resultat hätte geben können.

Ich möchte ferner hervorheben, dass ich Anfangs concentrirte, reine, nicht rauchende Salpetersäure vom specifischen Gewichte 1,4 verwendete und erst auf den gütigen schriftlichen Rath Gautier's bei den späteren Untersuchungen rauchende Salpetersäure zur Anwendung brachte.

Allein es waren auch die Untersuchungen bei zugegebenem Arsenik unter Anwendung reiner, nicht rauchender Salpetersäure angestellt worden und hatten trotzdem das schon erwähnte positive Resultat gegeben.

Ich konnte in der Litteratur der beiden letzten Jahre noch keine Mittheilung finden, welche sich mit der Nachprüfung von Gautier's Befunden beschäftigt hätte. Nur Gautier theilte mir mit, dass seine Resultate bereits mehrfach von Anderen Bestätigung erfahren hätten. Kurz erwähnen möchte ich nur eine Mittheilung der englischen Autoren E. Knecht und W. F. Dearden ${ }^{1}$ ) vom Beginn dieses Jahres, welche in einer allerdings auffallend geringen Menge $(5 \mathrm{~g})$ von Haaren normaler Individuen, die vorher kein Arsen genossen hatten, deutliche Spuren dieses Elementes nachweisen konnten. Diese Autoren geben aber nicht an, ob sie vorher ihre Reagentien auf absolute Arsenfreiheit geprüft haben.

Vielleicht ist auch die Möglichkeit nicht von der Hand zu weisen, dass die positiven Befunde Gautier's zum Theile in localen geologischen Verhältnissen begründet seien.

Ein Theil dieser Untersuchungen wurde im Laboratorium für medicinische Chemie des Herrn Hofrathes Professor E. Ludwig ausgeführt, welchem ich für sein gütiges Entgegenkommen meinen besten Dank ausspreche.

1) E. Knecht u. W. F. Dearden, The eliminat. of Arsenic trough the hair and its relation to arsen. poison, Lancet, 3. März 1901. 